\title{
La agricultura familiar agroecológica, una estrategia de desarrollo rural incluyente. Una revisión
}

\section{Agroecological family farming, an inclusive rural development strategy. A review}

\author{
Nelson Enrique Fonseca-Carreño ${ }^{1 *} \oplus$; Juan Davíd Salamanca-Merchan ${ }^{2 \oplus}$; \\ Zulma Yesenia Vega-Baquero ${ }^{3 \odot}$
}

Recibido para publicación: Septiembre 4 de 2018 - Aceptado para publicación: Mayo 24 de 2019

\begin{abstract}
RESUMEN
Dentro de la teoría de las ciencias ambientales, se menciona que existen interrelaciones complejas, dinámicas y constantes, que se establecen entre la agroecología, los ecosistemas y los territorios, en todas ellas la agricultura familiar adopta particular importancia como base de la subsistencia y la seguridad alimentaria. El objetivo de la presente investigación es determinar los planteamientos adyacentes a la Agricultura Familiar Agroecológica (AFA) y su constructo teórico. Este documento presenta una revisión sistemática de la agricultura familiar en bases de datos científicas, complementada con información bibliográfica depositada en revistas y libros científicos. Dentro de los hallazgos sobresale el hecho que la AFA ha sido un tema de interés, ya que subsidia su propia fertilidad y productividad, contribuyendo así, a la protección de la biodiversidad, dentro de las dimensiones económica, ecológica, política, social y cultural. Finalmente, se concluye que la AFA favorece la generación de buenas prácticas agroecológicas, demostrado a través de la presencia de alto número de especies tanto vegetales como animales y el conocimiento asociado a estas, contribuyendo a una mayor estabilidad en el uso del capital natural, aportando así a la sustentabilidad.
\end{abstract}

Palabras clave: Agroecología; Economía rural; Adaptabilidad; Cambio climático; Seguridad alimentaria; Sostenibilidad; Trabajo agrícola; Resiliencia; Agricultura campesina.

\footnotetext{
${ }^{1}$ Facultad de Ciencias Administrativas, Económicas y Contables, Universidad de Cundinamarca. Fusagasugá, (Colombia).

${ }^{2}$ Facultad de Ciencias Administrativas y Contables. Universidad de Boyacá, (Colombia).

${ }^{3}$ Facultad de Ciencias Administrativas, Económicas y Contables, Universidad de Cundinamarca. Fusagasugá. Grupo de investigación TEMCON.

*Autor para correspondencia: Nelson Fonseca

Email: nefonseca@cundinamarca.edu.co
}

\begin{abstract}
Among theories of environmental sciences, it is mentioned that there are complex, dynamic and constant established interrelations, among agroecology, ecosystems and territories, and for all them family farming is of particular importance as fundamental for subsistence and food security. The objective of this research is to determine the adjacent approaches to Agroecological Family Farming (AFA) and its theoretical construct. This document presents a systematic review of family farming in scientific databases, supplied by bibliographic information stored in scientific journals and books. Among the findings, highlights the fact that the AFA has been a topic of interest since it supports its own fertility and productivity, therefore contributing to the protection of biodiversity within economic, ecological, political, social and cultural dimensions. Finally, it is concluded that the AFA favors generation of good agroecological practices, evidenced through the presence of high numbers of both plant and animal species and their associated knowledge, contributing to greater stability in the use of natural capital and promoting sustainability.
\end{abstract}

Key words: Agroecology; Rural economy; Adaptability; Climate change; Food safety; Sustainability; Agricultural work; Resilience; Peasant.

\section{Cómo citar}

Fonseca-Carreño, N., Salamanca-Merchan, J. y Vega-Baquero, Z. 2019. La agricultura familiar agroecológica, una estrategía de desarrollo rural incluyente. Temas Agrarios, 24(2):96-107. https://doi.org/10.21897/rta.v24i2.1356

Temas Agrarios 2019. Este artículo se distribuye bajo los términos de la Licencia Creative Commons Attrubution 4.0 (https://creativecommons.org/licenses/by-nc/4.0/deed.es), que permite copiar, redistribuir, remezclar, transformar y crear a partir del material, de forma no 


\section{INTRODUCCIÓN}

Los sistemas de producción agropecuaria responden en su mayoría a características tradicionales de la producción con una fuerte incidencia familiar, respondiendo a factores externos como falta de capital, escasa tecnología, distorsiones en el mercado y condiciones climáticas adversas (Organización de las Naciones Unidas para la Agricultura y la Alimentación [FAO], 2014). Se percibe un fuerte vínculo entre los pobladores rurales con las actividades agropecuarias a través de un nexo entre los recursos disponibles (tierra, agua, biodiversidad), en efecto, las familias agrícolas rurales destinan mediante usos del suelo diferentes esquemas o distribuciones agrícolas/pecuarias según las épocas del año y de acuerdo a los ciclos climáticos (Albarracín-Zaidiza et al., 2019).

La Agricultura Familiar [AF] se caracteriza por poseer una escala de producción pequeña y altamente diversificada, al igual que de transformación de bienes y servicios, que se destinan para el autoconsumo o la venta (Cedrssa, 2014), como principal generadora de empleo e ingresos para la población rural (Ikerd, 2016). Lo anterior, permite a la $A F$, incrementar la eficiencia en el uso de los recursos endógenos, incentiva la incorporación de prácticas agroecológicas, apropia tecnologías para el aprovechamiento de las fuentes renovables de energía y contribuye gradualmente al logro de la soberanía alimentaria (CasimiroRodríguez y Casimiro-González, 2017).

La Comunidad Andina de Naciones [CAN], (CAN, 2011), propuso el concepto de Agricultura Familiar Agroecológica [AFA], por la dinámica de utilizar la mano de obra familiar, fuentes renovables de energía, recursos locales para el manejo agroecológico sin el uso de productos químicos, produciendo alimentos e ingresos para el desarrollo, fortaleciendo entre generaciones y la cultura agroecológica específica de un espacio predial.
En este contexto, Domínguez (2015), afirma que la AFA utiliza mano de obra familiar, la cual se basa en los conocimientos locales (Blandi et al., 2016), los cuales se trasmiten de padres a hijos con patrones culturales educativos como pilares de un proceso de desarrollo rural (Van der Ploeg, 2013) y, que para Casimiro-Rodríguez (2016), son energéticamente más eficientes y llegan a producir hasta diez veces más energía de la que consumen, lo cual fortalece su identidad, valores espirituales y culturales.

De igual forma, desarrolla conocimientos propios de la ocupación agropecuaria y se apoya en redes familiares y comunitarias (FAO, 2014), donde se preserva, valora y fomenta la multifuncionalidad de los métodos de producción campesina e implementa estrategias para la adaptación a posibles perturbaciones (Turbay et al., 2014), incrementando su resiliencia (capacidad del agroecosistema para mantener la productividad en presencia de estrés o perturbación), logrando así, mantener prácticas ancestrales que corresponden a enfoques agroecológicos (Prieto, 2011).

Dichas prácticas, las emplean para satisfacer las necesidades hídricas de los sistemas de producción (FAO, 2014), preservar la fertilidad y estructura del suelo (Ugas, 2014; Raigon, 2014), estimular las rotaciones agrícolas (Van der Ploeg, 2013) y promover principios ecológico-productivos incorporando una visión agroecológica (Vásquez y Martínez, 2015), a través del manejo y conservación de semillas y variedades cultivadas (recursos filogenéticos) (Casas y Moreno, 2014).

La presente revisión pretende dar a conocer algunos aspectos claves de la AFA, dentro del contexto estratégico del desarrollo rural con miras a procesos inclusivos, comenzado con la descripcióndelaevolucióndelaagriculturadesde lo convencional hasta llegar a la condensación de la denominada Agricultura Familiar, 
Fonseca-Carreño et al. - La agricultura familiar agroecológica.

recalcando la importancia que esta tiene desde el contexto global y centrándose en lo particular.

\section{De la agricultura convencional a la agricultura familiar agroecológica}

La agricultura ha sido clasificada de diversas formas a lo largo del tiempo, por ejemplo, INEGI (2008) la categoriza por la dependencia de agua, riego y humedad, Sáez (2009), lo hace por el tamaño de la producción de subsistencia y agroindustria y la FAO citado en SAGARPA (2012), es catalogada por el rendimiento y utilización de medios de producción intensivo y extensivo, así como por el modo de uso de la tierra en agricultura ecológica, tradicional, convencional, industrial, natural y de conservación.

Por tanto, la agricultura convencional se caracteriza por aplicación intensiva de sustancias de síntesis química, mecanización inadecuada de suelos, tala y quema de bosques (Paleologos et al., 2017). Otro elemento determinante lo constituye el régimen de tenencia de la tierra, sea en condiciones de aparcería, arrendamiento o propiedad, por mencionar algunas; ya que, como lo menciona León (2010), ello ejerce una significativa influencia sobre la base de sustentación ecosistémica y las relaciones sociales de producción.

Es así, que la agricultura convencional, está dada principalmente por sistemas de producción de monocultivos (sistemas productivos transitorios), por encima de cualquier otro arreglo de cultivo, funcional a un modelo de eficiencia orientado al mercado (León, 2010), con uso de prácticas agropecuarias convencionales basadas en el modelo de revolución verde, especialmente el uso intensivo de sustancias de síntesis química. Adicionalmente, la presencia de fenómenos agravantes como la ampliación de la frontera agrícola, a través de prácticas como la remoción de la cobertura vegetal natural (mediante tala y/o quema), fragmentación, desecación de humedales, inundación, remoción del barbecho, aumento de los procesos migratorios y la vulnerabilidad económica de la familia rural; así como la reducción significativa de la autonomía campesina, polarización de sociedades agrarias, acaparamiento de semillas e incluso agravamiento de la pobreza rural (León, 2010).

Bermúdez, Arenas y Moreno (2017) identificaron prácticas negativas respecto al manejo ambiental a las quemas de residuos sólidos, vertimientos domésticos, deforestación, contaminación de rondas hídricas, compactaciones de suelo y lodazales. Carreño y Baquero (2019), mencionan que uno de los mayores desafíos es disminuir o eliminar el uso de insumos de síntesis química pero no se trata de reemplazar un paquete químico por otro ecológico, lo que se busca es lograr cambios en el diseño y manejo de los agroecosistemas, para fortalecer los procesos ecológicos que brinda la biodiversidad presente. Es evidente, que los sistemas actuales de producción basados en el monocultivo tendrán que adaptarse para enfrentar estas presiones cambiantes asociadas a la frecuencia e intensidad de las condiciones meteorológicas extremas (Nicholls et al., 2015).

Conocer las características de los ecosistemas y cómo los mismos son afectados por ciertas prácticas agropecuarias, es esencial para planificar un manejo adecuado de los recursos (Sarandón y Flores, 2014) e interacciones que favorecen el cumplimiento de los procesos ecológicos de la agricultura convencional (Stupino et al., 2014). Así, la agroecología se convierte en un instrumento indispensable para diseñar fincas sustentables (Paleologos et al., 2017). Dussi y Flores (2018), afirman que deben ser económicamente viables, ecológicamente adecuados y socialmente aceptables. Hart (1985), mencionó que las fincas de 
Fonseca-Carreño et al. - La agricultura familiar agroecológica.

las AFA funcionan como una unidad de producción dentro del sector agrícola de un territorio, por ende, es la unidad básica de producción agropecuaria en la que interactúan factores bióticos, físicos y socioeconómicos, y es manejada generalmente por una familia que toma todo tipo de decisiones que afectan los procesos desarrollados en el predio (Ángel, 2016). De ahí que, la AFA subsidia su propia fertilidad y productividad, (Rosset y Martínez, 2013), contribuyendo así, a la protección de la biodiversidad, dentro de las dimensiones económica, ecológica, política, social y cultural (FAO, 2014), permitiendo generar lo que Altieri y Toledo (2011), a través de Crowley (2013), definen como una "agricultura sostenible".

La Secretaría General de la Comunidad Andina de Naciones [CAN], menciona que la finca como unidad de medida, puede presentar mayor productividad que otros sistemas agrícolas, debido a una mejor fertilidad, estabilidad del suelo, optimización del uso del agua, diversificación de cultivos, rendimientos en condiciones adversas y la generación de nuevos mercados (CAN, 2011); además, contribuye a restablecer, recuperar y mantener la biodiversidad a partir de la capacidad natural del suelo, plantas y animales (Chiriboga, 2012).

En efecto, la AFA se concibe como actividades agrícolas operadas principalmente por la mano de obra familiar (FAO, 2014). Al respecto, el Foro Mundial Rural celebrado en el año 2010, expresa que la AFA representa un valor simbólico y estratégico por sus funciones económicas, sociales, culturales, ambientales y territoriales. Este mismo foro, plantea, que más que un modelo de economía agraria, es la base de la producción sostenible de alimentos para avanzar hacia la seguridad alimentaria (Casimiro-Rodríguez y CasimiroGonzález, 2017). Para lo cual, genera una transformación y adaptación de los sistemas convencionales de producción, hacia sistemas de base agroecológica, que comprende no solo elementos técnicos, productivos y ecológicos, sino también aspectos sociales, culturales y económicos del productor rural (Acevedo, 2013); por tanto, se debe entender como un proceso multilíneal del cambio que ocurre a través del tiempo (Vázquez, 2015).

De esta forma, aunque pueda resultar compleja una transición desde actividades agropecuarias tradicionales hacia actividades agroecológicas, los resultados de la revisión permiten establecer que los beneficios para las familias productoras por dicha transición no solo son económicos, sino que también aportan valor ecológico a sus predios y permiten fortalecer lazos comunitarios construyendo así sociedades resilientes, sostenibles y saludables. Lo anterior, implica no solo la adopción de la normatividad legal vigente, sino también un cambio en la forma de producción, generando cambios positivos en el uso del suelo, el bienestar animal, la eficiencia del recurso hídrico, la reforestación de áreas boscosas y la integración de hábitats y dinámicas del entorno (Carreño y Baquero, 2018).

\section{Importancia de la agricultura familiar agroecológica AFA}

La AFA ha sido un tema de interés desde ámbitos económicos (Yúnez et al., 2013), de políticas públicas (BID, 2007; FAO, 2014), de desarrollo sostenible (Wymann et al., 2013) y por supuesto académico. Entorno a ella, se han abordado temas de acceso a mercados, cadenas de valor, políticas públicas, cambio climático, género, seguridad alimentaria y el incentivo a las familias como fuerza de trabajo; conteniendo acciones que representan el desarrollo incluyente y actividades en las que se reduce la pobreza (PNUD, 2016).

En las principales agendas de política internacional como la Agenda 21, Objetivos de Desarrollo del Milenio y más recientemente Objetivos del Desarrollo Sostenible, impulsadas por la Organización de Naciones Unidas se 
Fonseca-Carreño et al. - La agricultura familiar agroecológica.

deja de lado las diferentes formas a través de las cuales puede construirse cohesión social e intercambio de conocimientos a partir de la AFA. De ahí la importancia que reviste no solo la regulación sino también el fomento a través de políticas públicasyleyes, para la implementación de buenas prácticas agropecuarias, a fin de compatibilizar las actividades productivas con la protección ambiental, la cual contribuye a la seguridad alimentaria (Parmentier, 2014).

La FAO dentro del informe "El estado mundial de la agricultura y la alimentación", evidencia que hay más de 570 millones de sistemas de producción agrícola en el mundo, más del $90 \%$ de los sistemas dependen de mano de obra individual o familiar y provee la mayor cantidad de oportunidades de trabajo rural. A su vez menciona, que la AFA ocupa alrededor del $70 \%$ al $80 \%$ de las tierras agrícolas y producen más del $80 \%$ de los alimentos del mundo (FAO, 2014). Según estos criterios, la AFA es la forma de agricultura dominante en el mundo (Ángel, 2016).

Los sistemas de producción agrícolas han sido categorizados por distintas organizaciones en el mundo, los estudios realizados por la Comisión Económica para América Latina y el Caribe, el Instituto Interamericano de Cooperación para la Agricultura y la FAO (CEPAL, 2015), publicados en su documento "Perspectivas de la agricultura y del desarrollo rural en las Américas" señalan que los sistemas productivos que conciernen a la AFA sobrepasan el $75 \%$ de los países latinoamericanos y aporta más del $20 \%$ de la producción sectorial. Asimismo, la Comunidad Andina de Naciones (CAN, 2011) respecto a la AF en América Latina, menciona que esta constituye el $14 \%$ de la población total, genera entre $30 \%$ y $40 \%$ del PIB agrícola y más del $60 \%$ del empleo rural (genera empleo aproximadamente a dos de cada tres productores rurales). Al mismo tiempo, representa más del $80 \%$ de las unidades productivas, ocupa entre el $30 \%$ y el $60 \%$ de la superficie agropecuaria y forestal y es el principal abastecedor de la canasta básica de consumo de alimentos en todos los países (Ángel, 2016).

En el caso de Colombia en el año 2011, el Ministerio de Agricultura y Desarrollo Rural, conformó la Cadena Productiva de Agricultura Ecológica con su correspondiente línea de investigación y la constitución de consejos regionales para las diferentes regiones del país, lo que constituye un logro importante en cuanto a su visibilización y la posibilidad que se le asignen recursos para contribuir al desarrollo y consolidación, sobre todo a nivel de las comunidades campesinas (Ángel, 2016). Asimismo, se incluye la AF en el Plan Nacional de Desarrollo 2014-2018, donde resalta la inclusión de la AFA desde un enfoque de sostenibilidad ambiental (MADR, 2014).

Por tanto, la producción campesina y especialmente en Colombia se articula al sistema agroalimentario a través de: i) autoconsumo familiar y autoconsumo local a través de redes de intercambio de alimentos; ii) abastecimiento directo de mercados locales y iii) abastecimiento masivo a los centros urbanos por medio de una amplia red de intermediarios rural-urbanos conectados con las centrales mayoristas (León et al., 2015).

\section{El enfoque agroecológico dentro de la AFA}

Epistemológicamente la Agroecología se basa en la ecología, termodinámica y teoría de sistemas, y determina sus fundamentos éticos y filosóficos por medio del pensamiento ecológico (Lucantoni et al., 2018), a su vez, surge como un nuevo paradigma y ciencia capaz de validar y generar conocimientos para la evaluación, diseño y manejo de agroecosistemas sustentables (Sarandón y Flores, 2014), generando principios ecológicos para el manejo de los sistemas alimentarios (Gliessman, Friedmann y Howard, 2019), 
Fonseca-Carreño et al. - La agricultura familiar agroecológica.

que consiste en articular los componentes del agroecosistema (cultivos, animales, árboles, suelos) de manera que haya interacciones temporales y espaciales (Altieri, 2010).

Diversos autores han presentado la agroecología como una ciencia que se funda en principios, no obstante, no existe un consenso alrededor de cuáles pueden ser dichos principios que permita avanzar en la consolidación de la AFA (Gómez et al., 2017) y en qué medida puede resultar una herramienta exitosa para el mundo rural y su salida de la pobreza (Maletta, 2011). De ahí que, Nicholls y Altieri (2019), consideran que la agroecología define los principios ecológicos necesarios para desarrollar sistemas de producción sustentables y postulan los siguientes principios para un manejo agroecológico: i) diversificación vegetal y animal a nivel de especies en tiempo y en espacio; ii) reciclaje de nutrientes y materia orgánica; iii) provisión de condiciones edáficas óptimas para crecimiento de cultivos, manejando materia orgánica y estimulando la biología del suelo; iv) minimización de pérdidas de suelo y agua, manteniendo cobertura del suelo, control de erosión y manejando el microclima y v) minimización de pérdidas por insectos, patógenos y malezas mediante medidas preventivas $y$ estímulo de fauna benéfica, antagonistas y alelopatía (Nicholls y Altieri, 2019).

Existen en la actualidad, diversos conceptos relacionados con la agroecología que distan según cada autor, por ejemplo, para Bello, Jordá y Tello (2010), se entiende como "una producción agraria basada en los principios de la ecología", para Flores y Sarandón (2004), "imita a la naturaleza". Asimismo, León y Altieri (2010), la describen como "la ciencia que estudia la estructura y función de los agroecosistemas", desde el punto de vista ambiental. Por otra parte, Altieri y Nicholls (2012), señalan que la agroecología permite aumentar el reciclado de biomasa, optimizar la disponibilidad y el flujo de nutrientes. Por esto, dichos sistemas generan estabilidad biológica, conservación de los recursos, equidad de género, productividad, con el fin de promover tecnologías de producción que tengan adaptabilidad ambiental (MolinaMurillo, 2016).

Dicha idea la refuerza Dussi y Flores (2018), los cuales mencionan que la agroecología es una disciplina científica que reúne, sintetiza y aplica conocimientos de diferentes ciencias afines, con una óptica holística, sistémica, así como un fuerte componente ético, para generar conocimientos, validar y aplicar estrategias para diseñar, así como manejar y evaluar agroecosistemas sustentables. Suárez (2014), sugiere que la agroecología debe estudiar el agroecosistema, involucrando producción de alimentos, fibras y materias primas a partir de factores tecnológicos, dotaciones de recursos naturales e inversiones de capital, también una serie de factores vinculados con las circunstancias en que se desenvuelven y con los efectos que ellas producen en las sociedades y en los ecosistemas (León et al., 2014).

Como resultado, un elemento característico dentro de la agroecología es el social, puesto que son las personas las partícipes y beneficiarias de esta simbiosis. Es así como se configura la Ilamada AFA, la cual se caracteriza por la dinámica de utilizar principalmente mano de obra familiar, fuentes renovables de energía, recursos locales y garantizar el diseño y manejo agroecológico sin el uso de productos químicos, y así producir la mayor cantidad de alimentos e ingresos para el desarrollo fortaleciendo, entre generaciones, la cultura agroecológica específica de un espacio predial.

\section{El agroecosistema como unidad de análisis}

Desde el punto de vista ambiental, León (2010), define a los agroecosistemas como "el conjunto de relaciones e interacciones que 
Fonseca-Carreño et al. - La agricultura familiar agroecológica.

suceden entre organismos", interactuando con componentes socioculturales de los agricultores (Sarandón y Flores, 2014), modificados para producir alimentos, fibra, combustible y otros productos para el consumo y procesamiento humano (Altieri, 2010).

Los sistemas productivos convencionales, particularmente los monocultivos (Vigouroux, 2011), se caracterizan por que en estos se explotan una o varias especies de plantas, con la utilización de tecnologías con predominio de mecanización (Vázquez, 2015) y el uso intensivo de agrotóxicos que causan externalidades negativas (Dussi y Flores, 2018), donde las interacciones y procesos ecológicos se debilitan y, en consecuencia, se hace necesario utilizar más cantidad de insumos externos al sistema (Swift et al., 2004). Lo anterior conlleva a posibles pérdidas económicas por la falta de producción o una insuficiencia de la misma para el sustento familiar. Por lo cual, Altieri y Nicholls (2013), postulan que los agroecosistemas son más resilientes que los sistemas de producción agrícola convencional, ya que son más sensibles a las complejidades de la agricultura local, los cuales tienen la habilidad para recobrarse, reorganizarse y evolucionar en respuesta a un estrés o disturbio externo (Molina-Murillo et al., 2017).

Como resultado, surge la necesidad de establecer estrategias y diseños para un eficiente y eficaz manejo del agroecosistema con los recursos localmente disponibles, de tal manera que se pueda optimizar los procesos ecológicos, potenciando las interacciones positivas y limitando las negativas. Por esto, el Programa de las Naciones Unidas para el Medio Ambiente en el año 2011, propuso un esquema caracterizado por la implementación de policultivos; mayor heterogeneidad del hábitat y complejidad de los agroecosistemas en pro de un sistema agrícola diverso y flexible. Por lo cual, la finalidad de los Agroecosistemas es fortalecer la capacidad y la habilidad que tienen los agricultores para potenciar, adoptar e implementar herramientas y mecanismos administrativos, evidenciar los niveles de organización y conocer el grado de participación en el desarrollo de actividades antrópicas y así poder efectuar una planificación del uso de la tierra; para proteger, almacenar y usar en forma eficiente el agua, multiplicar las prácticas de conservación de suelos e incrementar la cobertura vegetal (Carreño, 2019). De manera que, varios autores relacionan imparcialmente el agroecosistema a la finca, sin embargo, la decisión sobre qué considerar como agroecosistema es todavía difusa y no existe ningún consenso entre los estudiosos sobre cuál es la verdadera o por lo menos la mejor unidad de análisis (León et al., 2015). Como respuesta a lo anterior, se propone la siguiente clasificación, como lo evidencia la tabla 1.

Tabla 1. Propuesta para clasificación de los agroecosistemas, según su actividad o uso.

\begin{tabular}{|c|c|c|c|c|}
\hline Uso agrícola & Hortícolas & Frutícolas & Monocultivo & Cultivos asociados \\
\hline Uso pecuario & De carne & De leche & Doble propósito & \\
\hline Uso forestal & Silvícola & Silvopastoril & Agroforestal & Industrial: Madera \\
\hline Tenencia de tierra & Propietarios & Arrendatarios & Aparceros & Ocupación de hecho \\
\hline Superficie de finca & Latifundio & Propiedad mediana & Minifundio & Microfundio \\
\hline Tiempo & Transitorio & Semi permanente & Permanente & \\
\hline Intensidad de uso & Intensivo & Semi intensivo & Semi extensivo & Extensivo \\
\hline Sistema & Convencional & Orgánico & Ecológico & \\
\hline Relaciones económicas & Economíacampesina & Agricultura predial & Pequeña escala & Agroindustrial \\
\hline Grupos humanos & Indígenas & Afrodescendientes & Colonos campesinos & Agroindustriales empresariales \\
\hline
\end{tabular}

Fuente: Adaptado de León et al. (2015). 
Estos aportes contribuyen a una mejor comprensión de los agroecosistemas y establecen elementos base para caracterizarlos en sus diferentes aspectos: socioeconómico, técnico-productivo y ambiental (Ángel, 2016), identificando la clasificación, escala de valoración, y la relación entre sus componentes.

\section{CONCLUSIONES}

La percepción campesina de la AFA en los agroecosistemas, advierte la existencia de riesgos, los cuales está fuertemente asociados a los periodos de escasez de agua, que limita el desarrollo de la producción y ponen en peligro la estabilidad de la familia, estos periodos han venido siendo cada vez más marcados e impactando negativamente los ingresos familiares, en menor escala se asocia los efectos de las fuertes lluvias que generan derrumbes y dificulta la movilidad de productos. Asimismo, se presentan problemas de erosión y deforestación, debido a la presencia de zonas de ladera, colinas y áreas de reserva hídrica intervenidas por el aumento de la frontera agrícola

En conclusión, los conceptos de agroecología a través de las AFA, demuestran los mismos conflictos epistemológicos que se dan en los debates ecologistas y ambientalistas. Por un lado, existe la discusión para saber si existe la posibilidad de establecer una definición universal de la AFA, la cual es de gran importancia frente a las necesidades $y$ oportunidades de cada territorio. No obstante, la AFA como estrategia de desarrollo rural incluyente, contribuye en la conceptualización teórica, a través de los conocimientos autóctonos y valores culturales de las comunidades rurales, que favorecen la generación de buenas prácticas agroecológicas; lo cual preserva y mantiene una mayor biodiversidad, demostrada a través de la presencia de alto número especies tanto vegetales como animales, así como del conocimiento asociado a estas, expresado en los diferentes usos económicos, ecológicos, políticos, sociales y culturales. Por otro lado, la agroecología, de manera general, está a favor de un enfoque constructivista, con relación al equilibrio de especies animales y vegetales, calidad de agua, suelos y arreglo de cultivos, de ahí que, afecta todas las decisiones económicas de utilización de insumos, transporte, mecanización, sanidad vegetal, cosecha, poscosecha, calidad y atención a las demandas de los consumidores.

Por último, es claro que la agroecología requiere anticipar en la reconstrucción de una postura más reflexiva, donde se genere una constante revisión de los discursos teóricos, con el propósito de alcanzar objetivos académicos, socioeconómicos y ambientales comunes, que permita determinar su autenticidad, como práctica científica racional.

\section{Conflicto de Intereses}

Los autores declaran que es un trabajo orginal y no existío conflicto de intereses de ningún tipo en la elaboración y publicación del manuscrito.

\section{REFERENCIAS}

Acevedo, A. 2013. Escuelas de agroecología en Colombia la construcción del conocimiento agroecológico en manos campesinas. En Congreso Latinoamericano de agroecología (Altieri MA, Sarandón S, Morales CF, Funes F, Siura S, eds.). Lima, Perú: SOCLA. http://orgprints.org/25086/.

Albarracín-Zaidiza, J. A., Fonseca-Carreño, N. y López-Vargas, L. 2019. Las prácticas agroecológicas como contribución a la sustentabilidad de los agroecosistemas. Caso provincia del Sumapaz. Ciencia y Agricultura, 16(2), 39-55.

doi.org/10.19053/01228420.v16. $\underline{\text { n2.2019.9139 }}$ 
Fonseca-Carreño et al. - La agricultura familiar agroecológica.

Altieri, M. 2010. El estado del arte de la agroecología: revisando avances y desafíos. In T. León \& M. A. Altieri (Eds.), Vertientes delpensamientoagroecológico: fundamentos y aplicaciones, pp 77-104.

Altieri, M. y Toledo, V. 2011. The agroecological revolution in Latin America: Rescuing nature, ensuring food sovereignty and empowering peasants. Journal of Peasant Studies, 38(3), 587-612.

doi.org/10.1080/03066150.2011.58294 $\underline{7}$

Altieri, M. y Nicholls, C. 2012. Agroecología: única esperanza para la soberanía alimentaria y la resiliencia socioecológica. Agroecología. 7(2): 65-83.

Altieri, M. y Nicholls, C. 2013. Agroecología y resiliencia al cambio climático: Principios y consideraciones metodológicas. Agroecología. 8(1):7-20.

Ángel, D. 2016. Evaluación de servicios ecosistémicos generados en la agricultura familiar agroecológica campesina (AFAC) del centro del departamento del valle del cauca. (Tesis Doctoral). Universidad Nacional de Colombia.

Banco Interamericano De Desarrollo [BID]. 2007. Políticas para la agricultura familiar en América Latina y el Caribe - Resumen ejecutivo.

Bello, A., Jordá, C. y Tello, J. 2010. Biodiversidad y bienestar humano: el papel de la biodiversidad funcional. Ecosistemas. 16 (3): 69- 80.

Bermúdez, C., Arenas, N. y Moreno, V. 2017. Caracterización socio-económica y ambiental en pequeños y medianos predios ganaderos en la región del Sumapaz. U.D.C.A Actualidad y Divulgación Científica. 20(1), 199-208. http://www.scielo.org.co/pdf/rudca/ v20n1/v20n1a21.pdf

Blandi, M., Cavalcante, M., Gargoloff, N. y Sarandón, S. 2016. Prácticas, conocimientos y percepciones que dificultan la conservación de la agrobiodiversidad. El caso del cinturón hortícola platense, Argentina. Cuadernos de Desarrollo Rural. 13(78), 97-122. doi: doi.org/10.11144/Javeriana.cdr13-78. $\underline{\text { isCC }}$
Carreño, N. 2019. Caracterización de agroecosistemas campesinos en el municipio de cabrera en la provincia del Sumapaz-Cundinamarca. Pensamiento udecino, 3(1), 49-60.

Carreño, N. y Baquero, Z. 2018. Propuesta de indicadores para evaluar la sostenibilidad en agro ecosistemas agrícola ganaderos en la región del Sumapaz. Pensamiento udecino, 2(1).

Carreño, N. y Baquero, Z. 2019. Sostenibilidad como estrategia de competitividad empresarial en sistemas de producción agropecuaria. Revista Estrategia Organizacional, 8(1).

doi: $10.22490 / 25392786.3168$

Casas, A. y Moreno, A. 2014. Seguridad alimentaria y cambio climático en América Latina. Leisa. Revista de Agroecología. 30(4): 5-7

Casimiro-Rodríguez y Casimiro-González. 2017. Agricultura familiar a pequeña escala en la economía cubana. Rev Temas. 1 (1): 59-66.

Casimiro-Rodríguez, L. 2016. Bases metodológicas para la resiliencia socioecologica de fincas familiares en Cuba. Tesis de doctorado. Medellín: Universidad de Antioquia. Disponible en: https://3colibris.files.wordpress. com/2018/01/rodriguezl 2016 basesmetodolc3b3gicasresiliencia.pdf

Centro de Estudios para el Desarrollo Rural Sustentable y la Soberanía Alimentaria [CEDRSSA]. 2014. Elementos para la definición de la Agricultura Familiar. México, D.F.: CEDRSSA.

Chiriboga, M. 2012. Desafíos de la pequeña agricultura familiar frente a la globalización. Perspectivas Rurales Nueva Época.

Comisión Económica para América Latina $y$ el Caribe [CEPAL], Instituto Interamericano de Cooperación para la Agricultura [IICA]. 2015. Perspectivas de la agricultura y del desarrollo rural en las Americas: Una mirada hacia América Latina y el Caribe 2015-2016.

ht tps://ww w. cepal.org/es/ publicaciones/39023-perspectivas-laagricultura-desarrollo-rural-americasmirada-america-latina 
Comunidad Andina de Naciones [CAN]. 2011. Agricultura Familiar Agroecológica Campesina en la Comunidad Andina. Una opción para mejorar la seguridad alimentaria y conservar la biodiversidad. Secretaria General de la Comunidad Andina, pp. 54.

Crowley, E. 2013. Con el apoyo adecuado, la agricultura familiar puede contribuir al futuro del desarrollo rural sostenible. Revista para el Desarrollo Rural. https://www.rural21.com/home/

Domínguez, D. 2015. La Soberanía Alimentaria como enfoque crítico y orientación alternativa del sistema agroalimentario global. Revista Pensamiento Americano.

Dussi, M. y Flores, L. 2018. Visión multidimensional de la agroecología como estrategia ante el cambio climático. INTERdisciplina, 6(14), 129-153.

Flores, C. y Sarandón, S. 2004. Limitaciones del análisis éconómico neoclásico para evaluar la sostenibilidad de los sistemas agrícolas. Un ejemplo que compara sistemas hortícolas orgánicos y convencionales. Revista de Agricultura Sostenible. 24 (2), 77-91.

Gliessman, S., Friedmann, H. y Howard, P. H. 2019. Agroecology and Food Sovereignty. IDS Bulletin, 50(2).

https://doi.org/10.19088/1968-2019.120

Gómez, E., Ríos, O. y Eschenhagen, D. 2017. Propuesta de unos principios generales para la ciencia de la agroecología: una reflexión. Revista Lasallista de Investigación, 14(2), 212-219.

Hart, R. 1985. Conceptos básicos sobre agroecosistemas. Centro Agronómico Tropical de Investigación y Enseñanza. CATIE. Turrialba, Costa Rica.

Ikerd, J. 2016. Granjas pequeñas multifuncionales: esenciales para la sostenibilidad agrícola y la soberanía alimentaria mundial. Meta-Colombia. Seminario. Producción Tropical Sostenible; una herramienta de liderazgo para la Orinoquia. Colombia: Universidad de los Llanos.

Instituto Nacional de Estadística Geografía e Informática [INEGI]. 2008. Guia para la Interpretación de Cartografía Uso Potencial del Suelo. México.
León, T. 2010. Agroecología: desafíos de una ciencia ambiental en construcción En Vertientes del pensamientoagroecológico: fundamentos y aplicaciones. Sociedad Científica Latinoamericana de Agroecología - Universidad Nacional de Colombia, pp $53-77$.

León, T. y Altieri M. 2010. Enseñanza, investigación y extensión en agroecología: la creación de un programa latinoamericano de agroecología. En Vertientes del pensamientoagroecológico: fundamentos y aplicaciones. SOCLA, pp $11-52$.

León, T., Mendoza, T. y Córdoba, C. 2014. La estructura agroecológica principal de la finca (EAP): un nuevo concepto útil en agroecología. Rev Agroecología. 9(1): 55-66.

León, T., Sánchez, M., Rojas, L., Ortiz, J., Bermúdez, J., Acevedo, A. y Angarita, A. 2015. Hacia una historia de la agroecología en Colombia. Rev Agroecología. 10 (2): 39-53.

Lucantoni, D., Jiménez, A., Acuña, I., Morejón, M. y Castro L. 2018. Conversión agroecológica para la seguridad y la soberanía alimentaria de una finca familiar. Rev Cooperativismo y Desarrollo. 6 (1): 61-69.

Maletta, H. 2011. Tendencias y perspectivas de la Agricultura Familiar en América Latina. Documento de Trabajo $N^{\circ} 1$. Proyecto Conocimiento y Cambio en Pobeza Rural y Desarrollo. Rimisp, Santiago, Chile.

Ministerio de Agricultura y Desarrollo Rural [MADR]. Plan Nacional de Desarrollo 2014-2018. 2014. https://www.minagricultura.gov.co/ planeacion-control-gestion/Gestin/ Plan\%20de\%20Acci \%C 3\%B3n/ PLAN\%20NACIONAL\%20DE\%20 DESARROLLO \%202014\%20-\%20 2018\%20TODOS\%20POR\%20UN\%20 NUEVO $\% 20 P A I S . p d f$

Molina-Murillo S. 2016. Desarrollo verde e inclusivo en respuesta al cambio climático. Ambientico. 25(8): 24-9. 
Molina-Murillo, S., Barrientos, G., Bonilla, M., Garita, C., Jiménez, A.,, Madriz, M. y Valdés, S. 2017. ¿Son las fincas agroecológicas resilientes? Algunos resultados utilizando la herramienta SHARP-FAO en Costa Rica. Revista Ingeniería, 27(2): 25-39.

Nicholls, C. y Altieri, M. 2019. Bases agroecológicas para la adaptación de la agricultura al cambio climático. Research Journal, 11(1),

doi.org/10.22458/urj.v11i1.2322

Nicholls, C., Henao, A. y Altieri, M. 2015. Agroecología y el diseño de sistemas agrícolas resilientes al cambio climático. Rev Agroecología. 10(1), 7-31.

Organización de las Naciones Unidas para la Alimentación y la Agricultura [FAO]. 2014. El estado mundial de la agricultura y la alimentación.

http://www.fao.org/3/a-i4036s.pdf

Paleologos, M., Lermanó, M., Blandi, M. y Sarandón, S. 2017. Las relaciones ecológicas: un aspecto central en el rediseño de agroecosistemas sustentables, a partir de la Agroecología. Redes -Universidad de Santa Cruz do Sul. 22:92-115.

Parmentier, S. 2014. Enfoques agroecológicos: ¿qué, por qué y cómo? Bruselas: OXFAM. http://future offood.org/pdfs/ OXFAM 2014 Scaling Up Agroecological Approaches.pdf

Prieto, E. 2011. Desarrollo de la metodología de evaluación de sostenibilidad de los campesinos de montaña en San José de Cusmapa (Nicaragua).

http://oa.upm.es/9036/1/TFM. Esperanza Arn\%C3\%A9s..pdf

Programa de las Naciones Unidas para el Desarrollo [PNUD]. 2016. Desarrollo inclusivo.

http://www.undp.org/content/undp/en/ home/ourwork/povertyreduction/focus areas/focus inclusive development.html (Consulta: octubre 28, 2018)

Raigon, M. 2014. La alimentación ecológica: cuestión de calidad. Revista de Agroecología. 4(30):10-2.
Rosset, P. y Martínez, M. 2013. Movimientos Sociales Rurales y Diálogo de Saberes: Territorios, Soberanía Alimentaria y Agroecología. En: Soberanía alimentaria: un diálogo crítico. Conferencia Internacional Universidad de Yale. Primero, F. (ed). Policy. 1-29.

Sáez, D. 2009. La agricultura y su evolución a la agroecologia. Editorial Obra propia.

Sarandon, S. y Flores, C. 2014. La Agroecología: el enfoque necesario para una agricultura sustentable. Agroecología. Bases teóricas para el diseño y manejo de agroecosistemas sustentables. Editores: Sarandón, Santiago Javier y Flores, Claudia Cecilia.

http://hdl.handle.net/10915/37280

Secretaría de Agricultura, Ganadería, Desarrollo Rural, Pesca y Alimentación [SAGARPA]. 2012. Agricultura familiar con potencial productivo en México. http://www.sagarpa.gob.mx/programas2/ evaluacione-sexternas/lists/otros \% 20 estudios/attachments/42/agricultura\%20 familiar final.pdf

Stupino, S., Iermanó, M., Gargoloff, N. and Bonicatto, M. 2014. La biodiversidad en los agroecosistemas. Agroecología: bases teóricas para el diseño y manejo de agroecosistemas sustentables. Colección libros de cátedra. Editorial de la Universidad Nacional de La Plata. Capítulo, 5, 131-158.

h t t p ://sedici.un I p.edu.ar / handle/10915/37280

Suárez, A. 2014. Evaluación de la Ágrobiodiversidad en fincas campesinas agroecológicas y convencionales en el centro del departamento del Valle del Cauca, Colombia. (Tesis de Maestría). Universidad Nacional de Colombia. Palmira, Colombia, pp 146.

Swift, MJ., Izac, A. y van Noordwijk, M. 2004. Biodiversidad y servicios ecosistémicos en paisajes agrícolas: ¿estamos haciendo las preguntas correctas? Agricultura, ecosistemas y medio ambiente, 104 (1), 113-134.

Turbay, S., Nates, B., Jaramillo, F., Velez, J. y Ocampo, O. 2014. Adaptación a la variabilidad climática entre los caficultores de las cuencas de los ríos Porce y Chinchina, Colombia. Rev Investigaciones Geográficas. 85 (1).95112. 
Ugas, R. 2014. La agricultura ecológica nutre mejor al campo y a la ciudad. Revista de Agroecologia. 4(30): 8-9.

Van der Ploeg, J. 2013. Diez cualidades de la agricultura familiar. Revista de Agroecologia. 29 (4): 3-6.

Vásquez y Martínez. 2015. Propuesta metodológica para la evaluación del proceso de reconversión agroecológica. Rev Agroecología. 10(1): 33-47.

Vázquez. 2015. Diseño y manejo agroecológico de sistemas de producción agropecuaria. En Sembrando en Tierra Viva. Manual de Agroecología. Proyecto Tierra Viva. La Habana, pp 133-160.

Vigouroux, J. 2011. Biodiversity, evolution and adaptation of cultivated crops. Comptes Rendus Biologies, pp 450-457.
Wymann, V., Romeo, R., Vita, A., Wurzinger, M. y Kohler, T. 2013. La Agricultura de montaña es agricultura familiar: Una contribución de las zonas de montaña al Año Internacional de la Agricultura Familiar 2014. Roma, Italia: FAO, CDE, BOKU.

http://www.fao.org/3/a-i3480s.pdf

Yúnez, Cisneros. y Meza. 2013. Situando la agricultura familiar en México. Principales características y tipología. Serie Documentos de Trabajo N¹49. Grupo de Trabajo: Desarrollo con Cohesión Territorial. Programa Cohesión Territorial para el Desarrollo. Santiago, Chile: Rimisp. 